\title{
COMPARISON OF DYNAMIC STABILITY DURING WALKING AND RUNNING ON NONMOTORIZED CURVED TREADMILL ACCORDING TO CURVATURE RADIUS
}

\author{
SAYUP KIM, JONGRYUN ROH and JOONHO HYEONG \\ Human Convergence Technology Group \\ Korea Institute of Industrial Technology \\ Ansan 151-742, South Korea \\ YOUNGHO KIM* \\ Department of Biomedical Engineering, Yonsei University \\ Wonju 220-710, South Korea \\ younghokim@yonsei.ac.kr
}

Received 9 September 2016

Revised 14 February 2017

Accepted 11 June 2017

Published 6 December 2017

\begin{abstract}
It is generally believed that running on a curved surface is more unstable than running on a flat surface. In this study, the dynamic stability of locomotion on a nonmotorized curved treadmill (NMCT) with three curvature radii was compared with that on a motorized flat treadmill. Sixteen healthy adult men maintained four different self-paced speeds: slow walking, fast walking, jogging, and running. Significant differences were statistically verified using two-way repeated-measures analysis of variance (ANOVA) according to the curvature radii and speeds, and the interaction effects were confirmed. Furthermore, to understand the significant differences between the speed and curvature radius, post hoc analyses were performed using one-way ANOVA. Except for the step width, the other parameters showed differences and correlation effects between the curvature radius and speed. As the curvature radius decreased, the stability decreased at slow speeds (slow walking) but increased at fast speeds (running). However, as the curvature radius increased, the stability increased at slow speeds (slow walking) but decreased at high speeds (running). The study results will help in suggesting the appropriate curvature radius for different user types such as athletes, the elderly, and people who require rehabilitation and will serve as preliminary data for designing the curvature radii of NMCTs.
\end{abstract}

Keywords: Curved treadmill; nonmotorized treadmill; curvature radius; dynamic stability; margin of stability.

*Corresponding author.

This is an Open Access article published by World Scientific Publishing Company. It is distributed under the terms of the Creative Commons Attribution 4.0 (CC-BY) License. Further distribution of this work is permitted, provided the original work is properly cited. 


\section{S. Kim et al.}

\section{Introduction}

A motorized flat treadmill (MFT) is a machine with an electrically rotating belt on which users can perform exercises such as walking or running. ${ }^{1,2}$ The MFT has the advantage of allowing users to perform aerobic exercises within small indoor areas by using its options to change the belt speed and inclination and thereby simulate different conditions involved in ground exercises. Currently, nonmotorized curved treadmills (NMCTs), which are based on a new concept, are under development. These treadmills do not require a motor, and their speed can be controlled freely by the user. The NMCT allows repetitive motions such as walking and running on a concavely curved belt. ${ }^{3,4}$ Additionally, unlike an MFT, acceleration and deceleration can be realized by simply changing the center of gravity of a body without requiring any other methods of control. Furthermore, users can run according to their individual capacities because the NMCT does not have a speed limit. ${ }^{3}$

The biomechanical and physiological advantages of NMCT workouts have been reported in many studies. The amount of calories burnt during NMCT workouts are higher than that burnt during MFT workouts because during NMCT workouts, the user has to physically generate the force required for belt rotation. ${ }^{3}$ However, the stress on the knees is small because the ground reaction forces are small. ${ }^{5}$ Furthermore, to maintain a steady speed on an NMCT, motor coordination between muscles and nerves is required, and thus, NMCT workouts are expected to significantly help rehabilitation as well. ${ }^{3}$ An NMCT can also be used to evaluate and improve exercising capabilities. It can also calculate the acceleration for every step of the user in real time and thus can be used for performing reliable sprint tests. ${ }^{6}$ However, NMCT workouts are different from the conventional, familiar MFT workouts, ${ }^{7}$ and the drawback of exceeding one's capacity subconsciously during a workout has been reported. ${ }^{5}$

NMCTs have different curvature radii depending on the manufacturer and product, whereas MFTs simply have flat running surfaces. Therefore, during training exercises on NMCTs, the physiological characteristics may vary because of differences in the curvature radii between different manufacturers and products. So far, studies on NMCTs have mostly focussed on the differences in the physiological effects between NMCT workouts and MFT workouts or ground workouts. Very few studies have considered how a change in the curvature radius affects the physiological and biomechanical characteristics. In particular, the curvature radius can significantly affect the exercise quality experienced by the user because the belt on an NMCT is supported by a number of bearings that form a curve and hence the rotating resistance is small. In addition, studies conducted so far to prove the workout capabilities and clinical results of NMCTs have the limitation of focussing on only a certain speed while analyzing running exercises. ${ }^{2,4,5,7}$ Even in cases in which different speeds are compared, only a single curvature radius is considered..$^{3,8}$ Thus, very few studies have considered the exercise characteristics and effects of NMCTs for various curvature radii and speeds. 
This study aimed to compare the dynamic stabilities of locomotion for various curvature radii and speeds. An NMCT test device that allowed the curvature radius to be switched to three different values was manufactured. The three curvature radii were used to compare the dynamic stabilities at various speeds and curvature radii and to compare NMCTs with MFTs, on which movements are performed on a flat surface. To compare the differences in exercise quality under various moving conditions, the margin of stability (MOS) was used to measure the dynamic stability that changes simultaneously with walking movements. ${ }^{9}$ However, the manner in which different curvature radii and speeds affect the MOS and the correlation between curvature radii and MFTs were unknown. Therefore, to determine how the magnitude of the curvature radius would affect the dynamic stability, this study formulated a hypothesis: the dynamic instability on NMCTs, which involve locomotion on a curved moving surface, would be greater than that on MFTs, which involve locomotion on a flat moving surface, because for NMCTs, the MOS in the anterior-posterior direction on the sagittal plane $\left(\mathrm{MOS}_{\mathrm{ap}}\right)$ was shorter and the MOS in the medial-lateral direction on the frontal plane $\left(\mathrm{MOS}_{\mathrm{ml}}\right)$ was longer. Furthermore, among the three curvature radii of the $\mathrm{NMCT}$, R3500, whose configuration was the closest to the MFT configuration, had a higher dynamic stability than R2000 and R2600.

\section{Methods}

\subsection{Subjects}

In this study, tests were performed on 16 healthy adult men in their $20 \mathrm{~s}$. The subjects were $24.4 \pm 1.0$ years old on average; their height was $174.1 \pm 4.4 \mathrm{~cm}$ on average and weight was $72.8 \pm 6.6 \mathrm{~kg}$ on average. The selected subjects did not have any critical physical musculoskeletal deformation or disorder of the nervous system, and no one who was highly obese with a body mass index of over $30 \mathrm{~kg} / \mathrm{m}^{2}$ was considered in the study. The Public Institution of Life Ethics Committee appointed by the Ministry of Health and Welfare in Korea approved the purpose and method of this study. Before performing tests, consent was obtained from the participants (IRB:P01-201605-11-001).

\subsection{Equipment: NMCT and MFT}

Figure 1 shows a conceptual diagram of both an NMCT with three adjustable curvature radii and a typical MFT. A frame was built for each curvature radius based on the commercial NMCT model SPT-1000C (Drax Corporation Ltd, Korea). The construction of the NMCT was such that the curved frame on the sides could be detached for changing the curvature radius. The minimum (R2000 mm) and maximum (R3500 mm) radii of products currently being sold commercially were chosen as the curvature radius range for the NMCT. Using the sequence of differences in the range R2000-R3500, four curvature radii were calculated: R2000, R2350, R2850, 


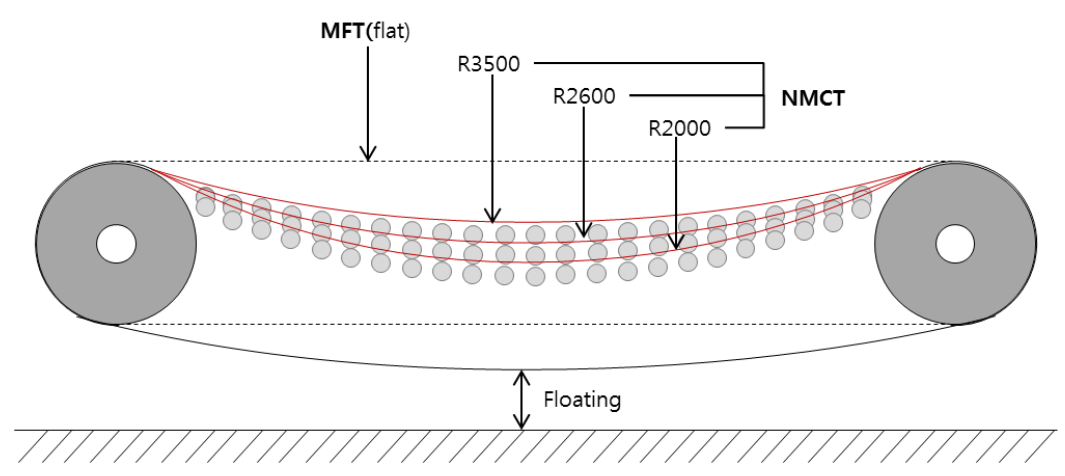

Fig. 1. Curvature radii of NMCT and MFT.

and R3500. Finally, three curvature radii were selected: R2000 mm, R2600 mm (the median value between R2350 and R2850), and R3500 mm. The height of the NMCT belt was chosen as the middle height between R2000 and R3500, with reference to the depth of concavity of R2600, which was the middle curvature radius. The distance between the wheels was different for each curvature radius, i.e., $\mathrm{R} 2000=1395 \mathrm{~mm}, \mathrm{R} 2600=1405 \mathrm{~mm}$, and $\mathrm{R} 3500=1415 \mathrm{~mm}$, with the maximum difference between the wheelbase values being $20 \mathrm{~mm}$. However, this difference was negligible considering the entire length of the wheelbase. The belt of the NMCT manufactured in this study showed natural sagging because of gravity; therefore, the NMCT was set up to float above the ground so that the belt did not drag along the ground. Furthermore, in addition to the NMCT with different curvature radii, an MFT (RX9000, Drax Corporation Ltd, Korea) with a flat surface was used for comparison.

\subsection{Test protocol}

Eight motion capture cameras (Osprey, Motion Analysis Corporation Ltd, USA) were used to capture the locomotion of the subjects. Using the Helen Hayes full body marker set, 25 makers were attached to the body. Each marker was measured at a sampling rate of $60 \mathrm{~Hz}$.

The test procedures and methods for the NMCT and MFT are shown in Fig. 2. Before performing the actual test, cadence (steps/min) was measured for four speeds on five subjects during a pre-test. The four types of locomotion — slow walking, fast walking, jogging, and running — were performed at self-paced speeds. To ensure that the subjects had an accurate perception, natural language usage, namely, "pace suitable for a light walk (slow walking), pace suitable for power walking without running (fast walking), pace suitable for a light jog (jogging), and pace suitable for running that is faster than a jog (running)" was used to distinguish each speed. The results of pre-tests showed speeds of $117.1 \pm 13.3$ beats $/ \mathrm{min}$ (bpm) for slow walking, $138.2 \pm 10.8 \mathrm{bpm}$ for fast walking, $159.1 \pm 6.1 \mathrm{bpm}$ for jogging, and 


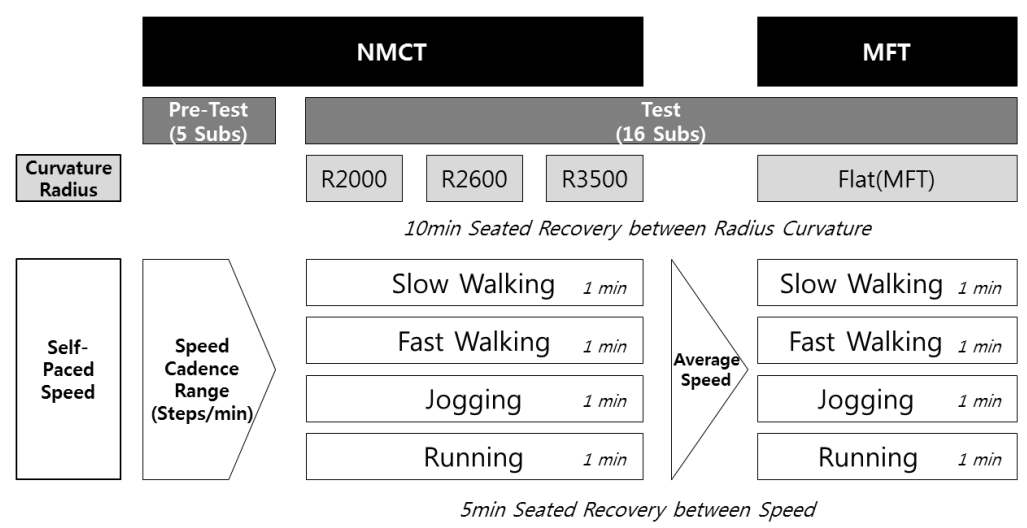

Fig. 2. Test protocol for NMCT and MFT.

175.7 $\pm 9.3 \mathrm{bpm}$ for running. The pre-test results confirmed that each speed, described using natural language, did not overlap with the other speeds during self-paced speed exercises on the NMCT. These results were used as an indicator to determine whether the subjects were running at the right speed during the actual test. For the actual test, the speed (self-paced speed, $\mathrm{km} / \mathrm{h}$ ) for each NMCT curvature radius was measured. Then, the average speed was calculated, which was applied to the MFT. Here, the average speed for the MFT was the average value of the speeds for the three curvature radii of the NMCT. The dynamic stability for exercises on the NMCT varied depending on the curvature radius, and the subjects might have been less familiar with exercising on an NMCT than on an MFT. ${ }^{7}$ Therefore, all the subjects were given a minimum of $10 \mathrm{~min}$ to adapt to the equipment. The curvature radius of the NMCT was randomly changed among the three values, and sufficient adjusting time was provided to the subjects for each curvature radius to minimize the differences arising from variations in the degree of familiarity. A minimum of 5 min of rest was given between exercises at each speed to avoid fatigue. Subjects tried to maintain stable movement as much as possible for each curvature radius at each speed. Furthermore, for consistency purposes, the subjects faced the front of the equipment and did not hold the handrail during movement.

\subsection{Data collection}

Movement motions for each curvature radius and speed were collected for 1 min using the Cortex program (Motion Analysis Corporation Ltd, USA) of the motion analysis system. One step was defined as the duration from the instant a right foot heel strike began to the instant a left foot heel strike occurred. From the start of movement, the most stable section between $20 \mathrm{~s}$ and $40 \mathrm{~s}$ containing 20 steps was selected as the analysis section. Full body segments were generated from the 25 markers attached to the body using the KinTools RT package of the Cortex program. The obtained results were used to calculate the center of mass (COM). 


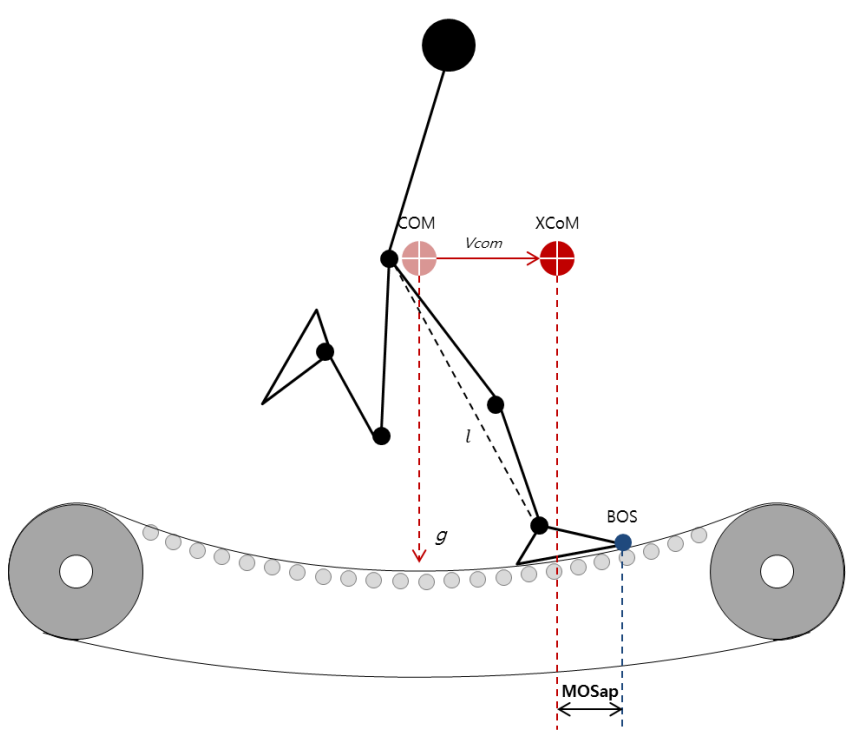

(a)

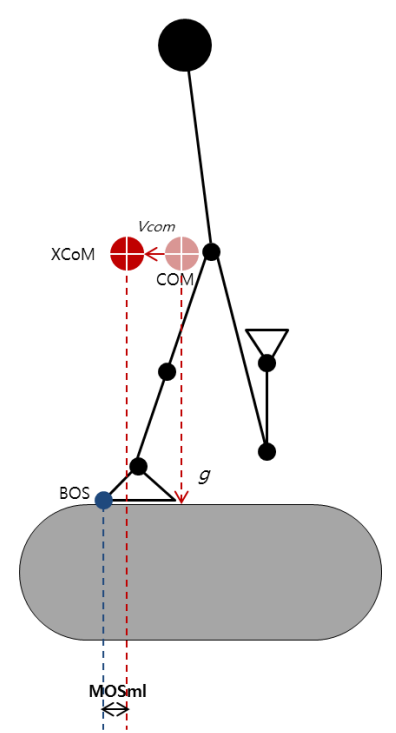

(b)

Fig. 3. MOS on NMCT: (a) $\mathrm{MOS}_{\mathrm{ap}}$ on sagittal plane and (b) $\mathrm{MOS}_{\mathrm{ml}}$ on frontal plane.

\subsection{Data analysis}

Figure 3 shows the definition of the MOS used in this study. The MOS results can be classified as either $\mathrm{MOS}_{\mathrm{ap}}$ or $\mathrm{MOS}_{\mathrm{ml}}$ according to the direction of motion. The location data for the toe and ankle markers attached on both the feet and the position data for the COM can be obtained. The following equations (Eqs. (1)-(3)) refer to the MOS calculations for each variable in order:

Base of support $(\mathrm{BOS})=$ Right or left foot position at heel strike,

$$
\text { Extrapolated center of mass }\left(X_{\mathrm{COM}}\right)=x+\frac{V \text { com }}{\omega 0} \text {. }
$$

Here, $V_{\mathrm{COM}}$ is the velocity of $\mathrm{COM}$ and $\omega_{0}=\sqrt{\frac{g}{l}}$, where $g$ is the gravitational acceleration $\left(9.81 \mathrm{~m} / \mathrm{s}^{2}\right)$ and $l$ is the equivalent pendulum length, i.e., the mean distance from the lateral heel marker to the COM at heel strike:

$$
\operatorname{MOS}=X_{\mathrm{COM}}-\mathrm{BOS}
$$

To prove the significant differences in the data according to the speed (slow walking, fast walking, jogging, and running) and curvature radius (R2000, R2600, R3500, and Flat) statistically $(P<0.001)$, the two-way repeated-measures analysis of variance (ANOVA) was applied to all the data using IBM SPSS Statistics V21.0 (IBM Corporation). Additionally, the interaction effect between the speed and 
curvature radius was verified $(P<0.001)$. Furthermore, one-way ANOVA was used for post hoc analysis $(P<0.05)$ and the significant differences for each specific value of the speed and curvature radius were identified.

\section{Results}

\subsection{Gait parameters}

\subsubsection{Step length $(\mathrm{mm})$}

The step length showed significant differences statistically according to the speed $(F=123.45, P<0.001)$ and curvature radius $(F=3.75, P<0.05)$. Furthermore, the step length showed interaction effects between the speed and curvature radius $(F=8.50, P<0.001)$. Post hoc analyses based on the speed [Fig. 5(a)] showed that for R2000 and R2600, the step lengths for fast walking and running were longer $(P<0.05)$ than those for slow walking and jogging. For R2000 and R2600, the step lengths for slow walking and jogging were similar $(P>0.05)$ to those for fast walking and running. However, as the curvature radius increased, the step length

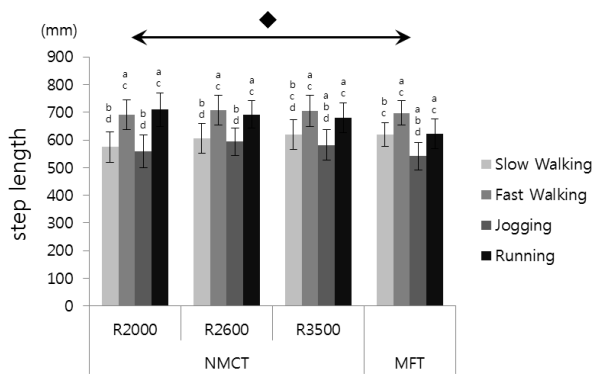

(a)

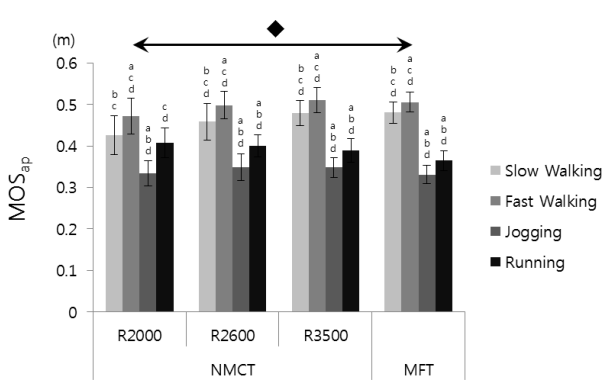

(c)

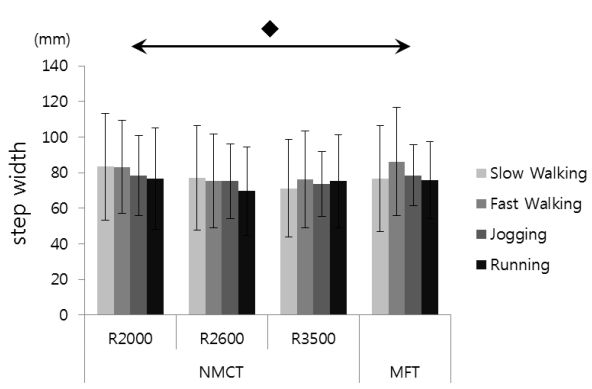

(b)

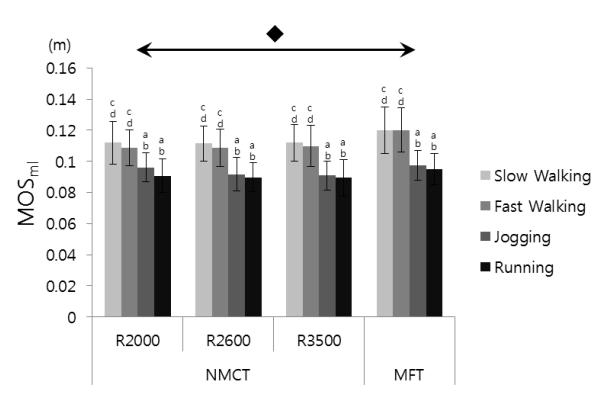

(d)

Fig. 4. Mean and standard deviation of step length (a), step width (b), $\operatorname{MOS}_{\mathrm{ap}}$ (c), and $\mathrm{MOS}_{\mathrm{ml}}$ (d) according to curvature radii. Significant differences in curvature radii $(P<0.001)$. Alphabet above the bar: significant differences resulting from post hoc multiple comparison are indicated. ( ${ }^{\text {a Significantly }}$ differs from slow walking, ${ }^{b}$ from fast walking, ${ }^{~}$ from jogging, ${ }^{d}$ from running. $)(P<0.05)$. 


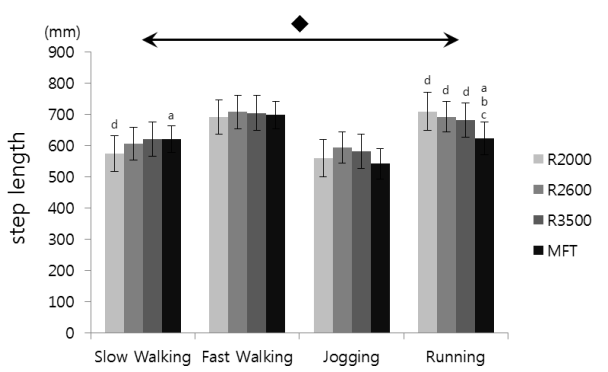

(a)

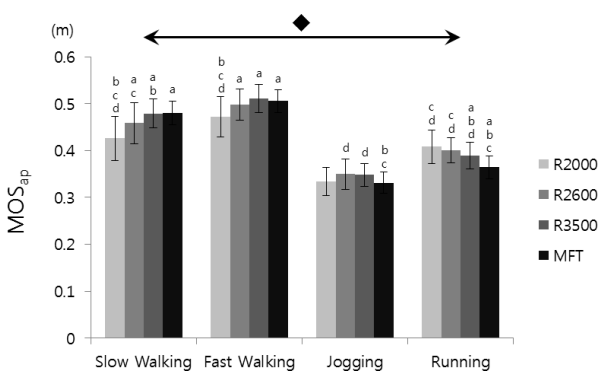

(c)

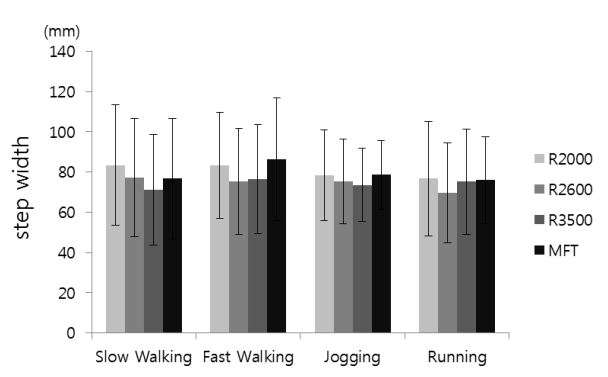

(b)

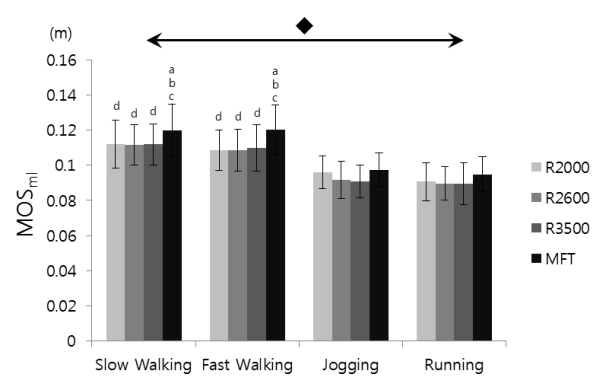

(d)

Fig. 5. Mean and standard deviation of step length (a), step width (b), $\mathrm{MOS}_{\mathrm{ap}}$ (c), and $\mathrm{MOS}_{\mathrm{ml}}$ (d) according to speeds. $\downarrow$, significant differences among the speeds $(P<0.001)$. Alphabet above the bar: significant differences resulting from post hoc multiple comparison are indicated ( ${ }^{\text {a }}$ significantly differs from R2000, ${ }^{\mathrm{b}}$ from R2600, ${ }^{\mathrm{c}}$ from R3500, and ${ }^{\mathrm{d}}$ from MFT $)(P<0.05)$.

for slow walking shortened and that for running lengthened. The step lengths for slow walking and running were similar $(P>0.05)$ for an MFT. Post hoc analyses based on the curvature radius [Fig. 4(a)] showed that the step length increased for slow walking as the curvature radius increased. However, significant differences were not found $(P>0.05)$. Conversely, as the curvature radius increased, the step length for running shortened and significant differences were found only for the MFT $(P<0.05)$. No significant effect of the curvature radius on the step length was found for fast walking or jogging, and no significant differences were found statistically either $(P>0.05)$.

\subsubsection{Step width $(\mathrm{mm})$}

The step width changed with the curvature radius $(F=3.58, P<0.05)$, and R2000 and the MFT flat surface had larger widths than R2600 and R3500 [Fig. 5(b)]. However, the step width did not change with the speed $(F=0.52, P>0.05)$ [Fig. 4(b)]. Furthermore, the step width had no interaction effects with either the speed or the curvature radius $(F=1.54, P>0.05)$, and post hoc analyses results also did not show significant differences for the speed or curvature radius $(P>0.05)$. 


\subsection{Margin of stability (MOS)}

\subsection{1. $\operatorname{MOS}_{a p}(m)$}

$\mathrm{MOS}_{\text {ap }}$ had significant differences statistically according to the speed $(F=423.54$, $P<0.001)$ and curvature radius $(F=7.80, P<0.05)$. Furthermore, MOS $_{\text {ap }}$ had interaction effects with the speed and curvature radius $(F=17.46, P<0.001)$. Post hoc analyses based on the speed [Fig. 5(c)] showed that $\mathrm{MOS}_{\mathrm{ap}}$ was the longest for fast walking and the shortest for jogging among all the curvature radii. Post hoc analyses based on the curvature radius [Fig. 4(c)] showed that $\mathrm{MOS}_{\mathrm{ap}}$ notably increased $(P<0.05)$ for slow walking as the curvature radius increased. Furthermore, $\mathrm{MOS}_{\mathrm{ap}}$ increased for fast walking as the curvature radius increased. However, a significant difference $(P<0.05)$ was found only for R2000. No differences in $\mathrm{MOS}_{\text {ap }}(P<0.05)$ were found between the curvature radii of the NMCT for jogging, excluding the MFT flat surface. For running, the results were the opposite for slow walking and fast walking. $\mathrm{MOS}_{\mathrm{ap}}$ increased as the curvature radius increased and showed significant differences for R3500 and the MFT flat surface.

\subsection{2. $\operatorname{MOS}_{m l}(m)$}

$\mathrm{MOS}_{\mathrm{ml}}$ showed significant differences statistically according to the speed $(F=74.65, P<0.001)$ and curvature radius $(F=9.43, P<0.001)$. Furthermore, $\mathrm{MOS}_{\mathrm{ml}}$ had interaction effects with the speed and curvature radius $(F=2.73$, $P<0.05)$. Post hoc analyses based on the speed [Fig. 5(d)] showed that $\mathrm{MOS}_{\mathrm{ml}}$ decreased $(P<0.05)$ as the speed increased for all the curvature radii values. Post hoc analyses based on the curvature radius [Fig. 4(d)] showed that $\mathrm{MOS}_{\mathrm{ml}}$ was the highest for slow walking and the lowest for running when the speed was the highest. However, post hoc analyses based on the curvature radius did not show any differences in $\mathrm{MOS}_{\mathrm{ml}}$ for different curvature radii at the same speed for the NMCT. However, slow walking and fast walking, which are both walking speeds, showed significant differences only for the MFT $(P<0.05)$.

\section{Discussion and Conclusions}

By comparing the effects of differences in the curvature radius and speed of an NMCT and the flat surface of an MFT, this study analyzed whether these differences affected the dynamic stability. It was assumed that the dynamic stability of the three curvature radii of an NMCT would decrease compared with the flat surface of an MFT and that consequently, $\mathrm{MOS}_{\mathrm{ap}}$ would decrease whereas $\mathrm{MOS}_{\mathrm{ml}}$ would increase. However, two results found in this study contradicted the speculation. First, $\mathrm{MOS}_{\mathrm{ml}}$ did not decrease with an increase in $\mathrm{MOS}_{\mathrm{ap}}$. As the curvature radius increased, $\mathrm{MOS}_{\mathrm{ap}}$ for slow walking and fast walking increased whereas that for running decreased. However, $\mathrm{MOS}_{\mathrm{ml}}$ did not show significant differences statistically for the curvature radius. Correlation analysis results for $\mathrm{MOS}_{\mathrm{ap}}$ and $\mathrm{MOS}_{\mathrm{ml}}$ 
showed relatively low correlation $(R=0.369)$. A comparison of these differences with previous study results indicated that changing the perturbation in the anteriorposterior direction changed $\mathrm{MOS}_{\mathrm{ap}}$ but did not change $\mathrm{MOS}_{\mathrm{ml}} \cdot{ }^{12}$ However, when the perturbation in the medial-lateral direction was changed, both $\mathrm{MOS}_{\text {ap }}$ and $\mathrm{MOS}_{\mathrm{ml}}$ showed significant differences statistically. ${ }^{10-12}$ In other words, in this study, the belt rotations on an NMCT and MFT affected the dynamic instability in the anterior-posterior direction; these results conformed with the study results of Ref. 12. Furthermore, the results of the present study showed that $\mathrm{MOS}_{\text {ap }}$ increased with an increase in the step length. These results were also comparable to the study results of Ref. 12, which indicated that $\mathrm{MOS}_{\text {ap }}$ increased with an increase in the step length, whereas $\mathrm{MOS}_{\mathrm{ml}}$ showed no change [Figs. 5(a) and 5(c)]. Correlation analysis results for $\mathrm{MOS}_{\mathrm{ap}}$ and the step length obtained in this study showed a relatively high correlation $(R=0.646)$, which agreed with the study results of Ref. 12 .

Secondly, it was predicted that dynamic stability would be higher for larger curvature radii at all speeds. However, statistically, no difference was found for jogging, and in fact, the dynamic stability decreased for running [Fig. 5(c)]. These anomalies might have been caused by differences between the exercise conditions; whereas exercises were performed relatively freely at a self-paced speed on the NMCT and exercises on the MFT required the user to adapt to the rotating belt powered by a motor (fixed speed). When only the three curvature radii of the NMCT were compared under the same self-paced speed condition, the effect of the speed on the dynamic stability was clearly observed. R2000, which was the smallest curvature radius, showed decreased dynamic stability compared with R2600 and R3500 for walking speeds (slow walking and fast walking). However, for running, R2000 had better stability than the other curvature radii [Fig. 5(c)]. However, R3500 showed high dynamic stability for exercises at walking speeds (slow walking and fast walking), although at increased speeds, R3500 had decreased stability compared with the other curvature radii [Fig. 5(c)]. These differences could have occurred because the performance of the equipment (NMCT) might not have satisfied the intentions of the runner. Whereas the runner might have intended to walk slowly, the low-resistance characteristic of the NMCT might have caused the belt to rotate fast. Alternatively, the runner might have intended to run fast, but the high resistance of the NMCT belt could have caused insufficient rotation. Such instances might have caused a decrease in the dynamic stability. This also meant that a mismatch between the supporting belt surface and user could increase the chances of falling. This indicated the necessity of suggesting exercises with an appropriate speed for a given curvature radius.

The present study results will be useful for selecting the curvature radius according to the intended use in the future. For example, products with a large curvature radius will be appropriate for rehabilitation and exercise for the elderly, who mostly require walking exercises. Products with a small curvature radius will be more appropriate for athletes who need to perform fast running exercises such as sprinting. Currently, many treadmill manufacturers are introducing NMCT 
products with different curvature radii. The present study results will serve as preliminary data for research on analyzing biomechanical characteristics for varying curvature radii.

\section{Acknowledgment}

This study has been conducted with the support of the Korea Institute of Industrial Technology as "Development of smart textronic products (KITECH JA-17-0045)".

\section{References}

1. Alton F, Baldey L, Caplan S, Morrissey MC, A kinematic comparison of overground and treadmill walking, Clin Biomech 13:434-440, 1998.

2. Franks KA, Brown LE, Coburn JW, Kersey RD, Bottaro M, Effects of motorized vs non-motorized treadmill training on hamstring/quadriceps strength ratios, J Sports Sci Med 11:71-76, 2012.

3. Smoliga JM, Hegedus EJ, Ford KR, Increased physiologic intensity during walking and running on a nonmotorized, curved treadmill, Phys Ther Sport 16:262-267, 2015.

4. Gonzalez AM, Wells AJ, Hoffman JR, Stout JR, Fragala MS, Mangine GT, McCormack WP, Townsend JR, Jajtner AR, Emerson NS, Robinson EH IV, Reliability of the Woodway curve non-motorized treadmill for assessing anaerobic performance, J Sports Sci Med 12:104-108, 2013.

5. Kashiwagi Y, Hirano T, Yamagishi M, Hakamada N, Kaneko KI, Funato K, Characteristics of ground reaction force and lower limb kinematics during running on selfdriven treadmill equipped with curve-shaped bed in sagittal plane, Jpn Soc Phys Fit Sports Med 64:333-344, 2015.

6. Fullenkamp AM, Laurent CM, Campbell BM, Automated gait temporal-spatial assessment from non-motorized treadmill belt speed data, Gait Posture 41:141-145, 2015.

7. Stevens CJ, Hacene J, Wellham B, Sculley DV, Callister R, Taylor L, Dascombe BJ, The validity of endurance running performance on the Curve $3^{\mathrm{TM}}$ non-motorized treadmill, J Sports Sci 33:1141-1148, 2015.

8. Tofari PJ, McLean BD, Kemp J, Cormack S, A self-paced intermittent protocol on a non-motorised treadmill: A reliable alternative to assessing team-sport running performance, J Sports Sci Med 14:62-68, 2015.

9. Hof AL, Gazendam MGJ, Sinke WE, The condition for dynamic stability, J Biomech 38:1-8, 2005.

10. Young PMM, Wilken JM, Dingwell JB, Dynamic margins of stability during human walking in destabilizing environments, J Biomech 45:1053-1059, 2012.

11. Hak L, Houdijk H, Steenbrink F, Mert A, van der Wurff P, Beek PJ, van Dieën JH, Speeding up or slowing down? Gait adaptations to preserve gait stability in response to balance perturbations, Gait Posture 36:260-264, 2012.

12. Young PMM, Dingwell JB, Voluntary change in step width and step length during human walking affect dynamic margin of stability, Gait Posture 36:219-224, 2012. 